\title{
Abscess in the Splenium of the Corpus Callosum Treated with Direct Drainage via an Occipital Interhemispheric Approach
}

\author{
Yasushi Motoyama1, Hisashi Kawai ${ }^{1}$, Yohei Kogeichi' ${ }^{1}$ Pritam Gurung ${ }^{2}$, Young-Soo Park1, \\ Hiroyuki Nakase ${ }^{1}$ \\ ${ }^{1}$ Department of Neurosurgery, Nara Medical University, Kashihara, Japan \\ ${ }^{2}$ Ohnishi Neurological Center, Akashi, Japan \\ Email: myasushi@naramed-u.ac.jp
}

Received 7 January 2015; accepted 22 January 2015; published 26 January 2015

Copyright (C) 2015 by authors and Scientific Research Publishing Inc.

This work is licensed under the Creative Commons Attribution International License (CC BY). http://creativecommons.org/licenses/by/4.0/

(c) (7) Open Access

\begin{abstract}
Lesions in the corpus callosum typically represent malignant tumors such as glioblastoma or lymphoma, because of its compact structure comprising tightly packed white-matter tracts. Brain abscess is rarely seen in the corpus callosum. To the best of our knowledge, solitary bacterial abscess confined to the splenium of the corpus callosum has not been reported previously. We report the case of a 72-year-old woman with rapidly progressing disturbance of consciousness following 1 week of antibiotic treatment for bacterial meningitis. Magnetic resonance imaging demonstrated a ring-enhancing round mass located in the splenium of the corpus callosum on gadolinium-enhanced T1-weighted imaging, also showing a bright signal on diffusion-weighted imaging. The patient underwent occipital craniotomy and direct drainage of the lesion in the splenium through the interhemispheric fissure and achieved complete recovery. Brain abscess should be considered among the differential diagnoses for lesions in the splenium of the corpus callosum. An occipital interhemispheric approach to the splenium might be an important option in cases of brain abscess.
\end{abstract}

\section{Keywords}

Brain Abscess, Splenium of Corpus Callosum, Microsurgical Drainage

\section{Introduction}

The corpus callosum consists of densely bundled white-matter tracts connecting the two cerebral hemispheres,

How to cite this paper: Motoyama, Y., Kawai, H., Kogeichi, Y., Gurung, P., Park, Y.-S. and Nakase, H. (2015) Abscess in the Splenium of the Corpus Callosum Treated with Direct Drainage via an Occipital Interhemispheric Approach. Open Journal of Modern Neurosurgery, 5, 34-40. http://dx.doi.org/10.4236/ojmn.2015.51006 
forming a compact structure that largely blocks interstitial edema and tumor spread. Accordingly, only aggressive tumors such as glioblastoma multiforme and malignant lymphoma typically cross or involve the corpus callosum. Isolated lesions of the corpus callosum are rare and may represent reversible responses to metabolic or myelination abnormalities, particularly in the splenium [1]-[5]. The corpus callosum is also a very uncommon site of brain abscess. Only a small number of reports have described abscess formation in the corpus callosum, including cases of tuberculoma [6], toxoplasmosis in a patient infected by human immunodeficiency virus (HIV) [7], and aspergillosis in immune-compromised patients being treated for malignancy with chemotherapy [8]. To the best of our knowledge, solitary bacterial abscess in the splenium of the corpus callosum without any other pathological condition has not been described previously.

Deep-seated abscess forming at locations such as the brainstem and basal ganglia is often treated with stereotactic aspiration [9]-[11]. When stereotactic surgery is performed for brain abscess, communication between the abscess and cerebrospinal fluid (CSF) spaces should basically be avoided to minimize the risk of disseminating the infective agent. The corpus callosum is located deep in the midline, but is surrounded by CSF spaces including the lateral ventricles, pericallosal cistern, and third ventricle.

We describe herein a case of solitary bacterial abscess in the splenium of the corpus callosum presenting with rapidly progressing disturbance of consciousness following treatment for bacterial meningitis. The patient was successfully treated with occipital craniotomy and direct puncture of the splenium under microscopic guidance to aspirate purulent contents. We also discuss the pathophysiology and surgical treatment strategy.

\section{Case Report}

A 72-year-old woman with no significant history, including of systemic infectious disease, developed severe headache and spiking fever. A local hospital diagnosed bacterial meningitis from the results of lumbar puncture, which revealed a cell count of 27,600 cells $/ \mathrm{mm}^{3}$, protein $>500 \mathrm{mg} / \mathrm{dl}$, and a glucose level of $50 \mathrm{mg} / \mathrm{dl}$ in CSF. On the basis of the clinical and laboratory findings, empiric treatment with ceftriaxone, vancomycin, and ampicillin was started. CSF culture showed positive results after incubation at 37 degree Celsius for 2 days. The isolates were subsequently identified as Streptococcus intermedius. The condition of the patient improved, but severe headache and spiking fever recurred 1 week later. Although broad-spectrum antibiotics had been administered, the patient showed a rapid deterioration in level of consciousness, so she was referred to our institution for further treatment 10 days after initiating chemotherapy. On arrival, the patient was restless and confused, showing meningeal irritation signs including nuchal rigidity, photophobia, and severe headache, along with a high fever of 39.2 degree Celsius. Magnetic resonance imaging (MRI) demonstrated a round mass in the splenium of the corpus callosum, showing ring-enhancement on T1-weighted imaging after gadolinium administration and signal hyperintensity surrounding the lesion on T2-weighted imaging suggesting perifocal edema (Figure 1(a) and Figure 1(b)). Contents of the cystic lesion were depicted as a bright signal on diffusion-weighted imaging (DWI) and as a low-intensity region on apparent diffusion coefficient (ADC) mapping, consistent with brain abscess (Figure 1(c)). The abscess appeared to show slight perforation leading into the right lateral ventricle from MRI findings, and a risk of rapid deterioration in neurological condition was thus considered present. Emergent surgical intervention was therefore performed on the day of transfer. After the spinal drainage catheter was inserted at the L3/L4 level, the patient was placed in the park bench position with right side down. The head was fixed in the dependent 45 degree oblique position. Right occipital craniotomy extending across the superior sagittal sinus (SSS) was conducted to open the dura mater close to the sagittal sinus. After craniotomy, CSF was released from the lumbar catheter to obtain a sufficient corridor in the interhemispheric fissure. The right occipital lobe was gently retracted while paying special attention to avoid excessive compression by the spatula. After the free margin of the falx cerebri and inferior sagittal sinus (ISS), tentorial incisura, and straight sinus were confirmed, the splenium of the corpus callosum was identified and punctured using a silicone catheter. Although no abnormalities on the surface of the splenium were apparent, viscous, yellowish, purulent material was drained from the inserted catheter. To avoid purulent contents spilling into the subarachnoid space, the silicone catheter for drainage was fixed before closing the surgical wound (Figure 2). The catheter was left in place until no purulent matter had been not discharged for 3 consecutive days (Figure 3). Administration of antibiotics continued for 8 weeks, even though her consciousness improved immediately postoperatively. MRI demonstrated complete remission of abscess as of 1 month after surgery (Figure 4(a) and Figure 4(b)). The patient was discharged in an ambulatory state with no neurological deficits. 


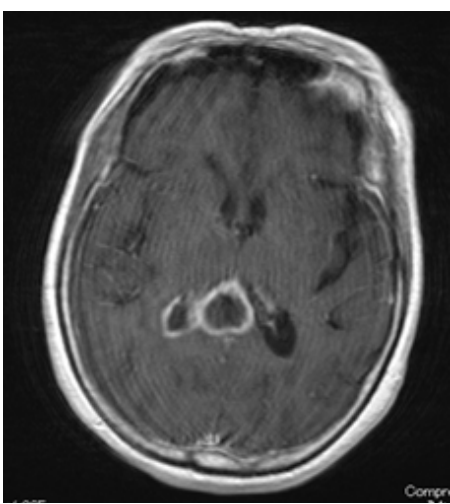

(a)

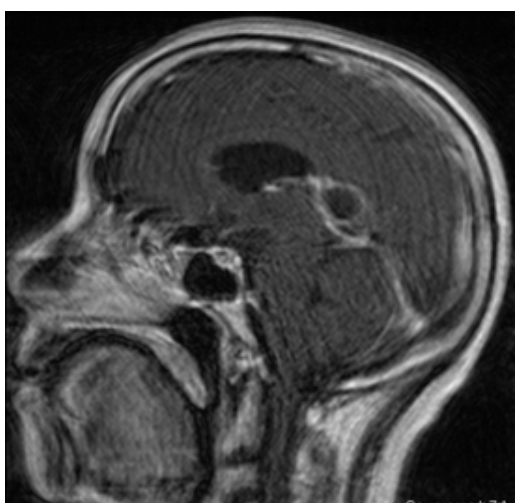

(b)

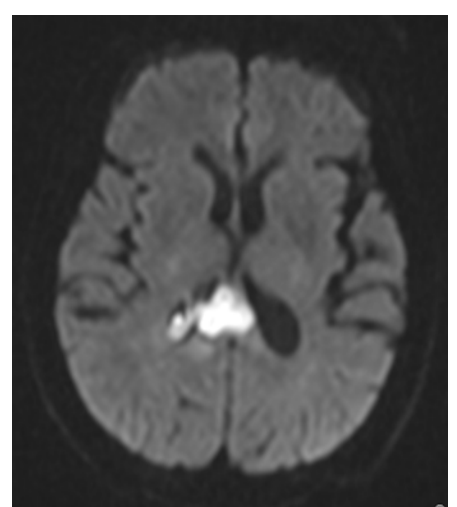

(c)

Figure 1. MRI showing a ring-enhancing round lesion in the splenium on T1-weighted imaging with Gd contrast. (a) Axial image demonstrating slight enhancement of the wall of the left ventricle in the posterior horn, suggesting ventriculitis due to perforation of the abscess; (b) Sagittal image indicating lesion location in the splenium; (c) DWI shows a bright signal for lesion contents.

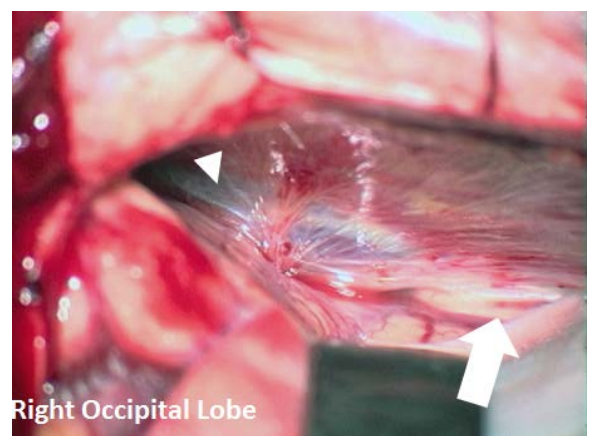

(a)

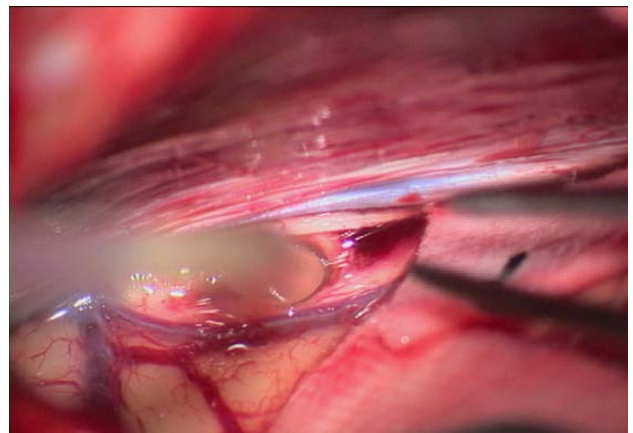

(b)

Figure 2. (a) Falx cerebri, straight sinus (white arrowhead), and splenium (white arrow) identified intraoperatively in the falcotentorial region; (b) Yellowish purulent matter is drained through the puncturing silicone catheter.

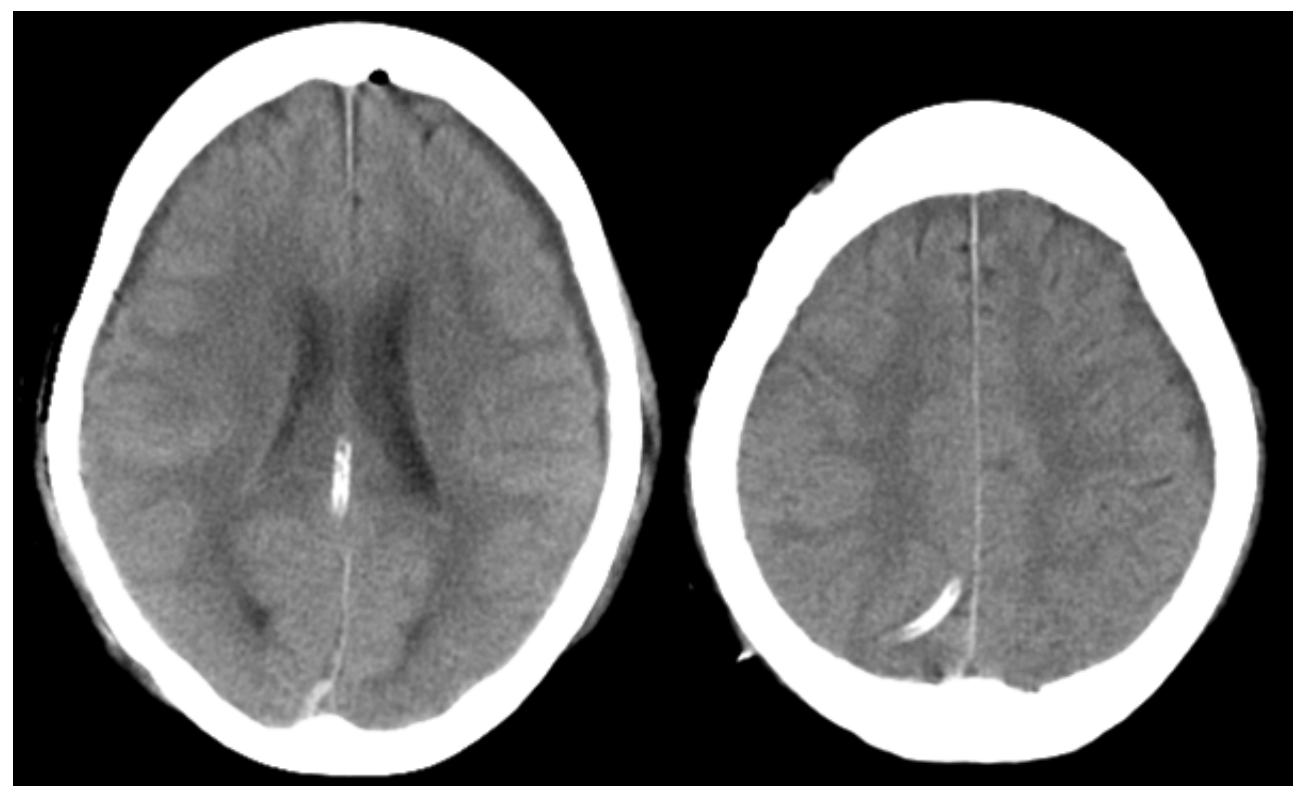

Figure 3. Postoperative computed tomography of the head shows the tip of catheter in the splenium of the corpus callosum through the occipital interhemispheric fissure. 


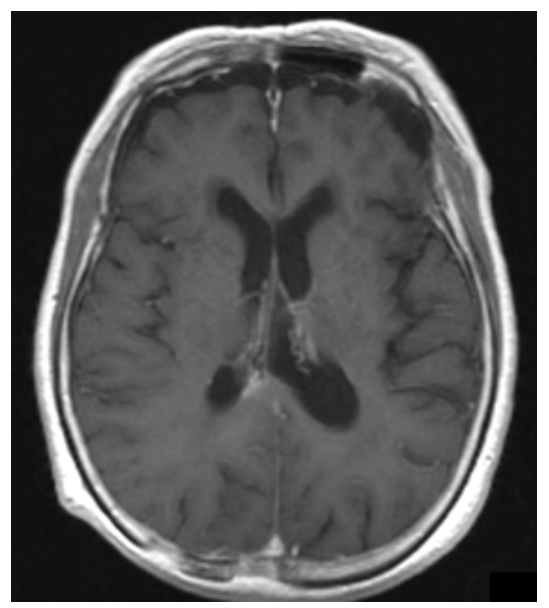

(a)

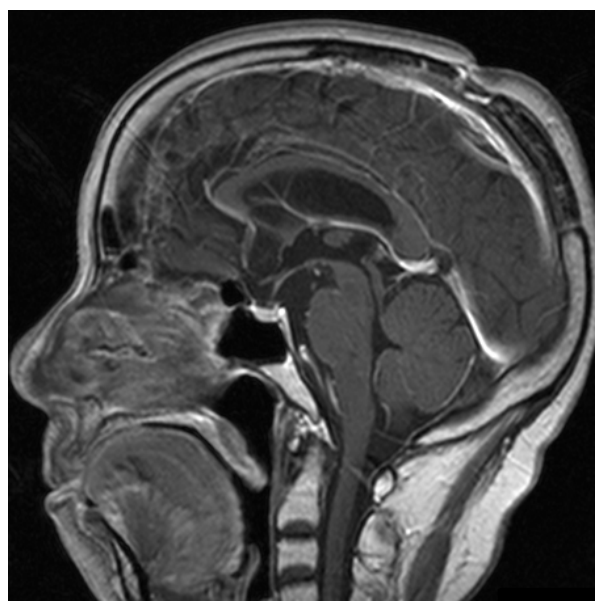

(b)

Figure 4. One month after drainage, MRI demonstrates complete remission of the abscess. (a) Axial image; (b) Sagittal image.

\section{Discussion}

The dense, compact nature of the white matter tracts in the corpus callosum, relative to the adjacent hemispheric white matter, make this structure a formidable barrier to both the flow of interstitial edema and tumor spread [12]. Accordingly, only aggressive tumors such as glioblastoma multiforme and lymphoma typically cross or involve the corpus callosum. When the corpus callosum is affected by glioblastoma multiforme, a characteristic bihemispheric involvement is commonly displayed, resulting in the classic butterfly pattern [13]. The corpus callosum is also included as a common location of lymphoma, along with deep gray matter structures and the periventricular region. Lymphoma differs from glioblastoma multiforme in that less peritumoral edema is present, and the lesions are more commonly multiple, less necrotic, highly radiosensitive, and frequently show dramatic temporary response to steroid administration, producing "vanishing lesions" [14].

In addition to such neoplastic lesions, the corpus callosum can be involved in a variety of pathologies, including demyelinating processes like multiple sclerosis, traumatic hemorrhage associated with the tight nature of the structure and the fixation to the dura, rendering it susceptible to shear stress, and lipoma as a congenital abnormality (mainly occurring in the region of the corpus callosum and pericallosal cistern, accounting for up to $65 \%$ of all intracranial lipomas and frequently associated with callosal dysgenesis) [15].

Infectious conditions seldom primarily involve the corpus callosum unless encephalitis or ependymitis occurs adjacent to and extends into the corpus callosum. Reinarz et al. reported on 11 patients with encephalitis evaluated by MRI and found only one with involvement of the corpus callosum [16].

Another report described a patient with HIV and toxoplasmosis demonstrating an irregular ring-enhancing lesion in the corpus callosum mimicking glioma with butterfly signs [7]. Immunocompromised patients are also at increased risk of fungal infection involving the CNS. Amongst the fungal pathogens, Candida and Aspergillus are the main organisms causing cerebral abscess. The respiratory system is the primary site for infection via inhalation of spores. The corpus callosum is reported to be included as a common site of Aspergillus infection along with the basal ganglia, thalami, brainstem, and gray-white matter junction [17].

In our patient, solitary abscess formation was confined in the splenium, even though ventriculitis due to perforation of the abscess was observed. In many cases of infection involving the corpus callosum reported previously, the lesion of the splenium was only one of multiple lesions or disseminations, or part of the invasive front. In our patient, the abscess was confined in the corpus callosum and not one of multiple foci. This type of abscess formation does not appear to have been described before.

Anatomically, the splenium is the posterior end of the corpus callosum, representing the thickest part, overlapping the tela chorioidea of the third ventricle and the mid-brain. In addition, bilateral posterior bodies of the lateral ventricles are close to the splenium.

Transient signal changes in the splenium have recently been reported in a wide variety of conditions, including seizures, antiepileptic drug toxicity or withdrawal [2] [3], sympathomimetic-induced kaleidoscopic visual 
illusions [18], hypoglycemia, hypernatremia, osmotic myelinolysis, Wernicke encephalopathy [4], and hemolytic uremic syndrome [19]. Lesions are well-circumscribed and located in the central splenium, with T2-weighted and fluid-attenuated inversion recovery hyperintense and T1-weighted hypointense signals. DWI demonstrated signal hyperintensity in the splenium with a low signal on ADC mapping. In the acute phase, contrast enhancement and reduced diffusivity may be seen. After disease recovery or withdrawal of the offending agent, imaging abnormalities resolve over several weeks to months [20] [21]. These lesions in the splenium have also been documented to be caused by various infective agents, such as influenza [22], rotavirus [23] [24], mumps, Escherichia coli, and adenovirus [25]. The changes become apparent on MRI from as early as the second day after symptom onset and CSF findings are usually normal or nonspecific. The pathophysiology is poorly understood, but is thought to reflect reversible demyelination and intracellular edema secondary to infectious, inflammatory, or metabolic disturbances [21] [26] [27].

Our hypothesis to explain why the lesion in this patient was confined to the splenium is as follows. In this patient, meningitis had been treated with antibiotics for 10 days before detection of the splenial abscess. If some reversible changes such as demyelination or intracellular edema due to antecedent bacterial meningitis had occurred in this patient, the central part of the splenium contiguous with CSF might have been more susceptible to infective agents than usual. The splenium might thus have become a focus of bacterial infection.

In the future, with the evolution of antibiotic-resistant bacteria, diversification of disease, and the prevalence of MRI, formation of splenial abscesses as in this case may be encountered more often.

Deep-seated abscesses are often treated with stereotactic aspiration [9]-[11]. However, stereotactic aspiration of the abscess may be unsuccessful if the abscess is multiloculated or if the pus is very thick. Such methods can also induce hemorrhage from the abscess capsule during penetration. Occasionally, excessive suction can result in additional bleeding from surrounding parenchyma, because assessment of complete evacuation is very difficult.

In addition, the corpus callosum is located in the midline and surrounded by subarachnoid cisterns and ventricles. If the suspected infectious lesion located in the splenium had been treated by stereotactic aspiration, a linear trajectory to the lesion that avoided communication of the abscess with ventricles or subarachnoid spaces would be difficult.

Moreover, even though modern diagnostic imaging techniques have started to reveal not only the anatomy of cerebral lesions, but also their molecular basis, differentiation of abscess from other lesions is not always possible. In such cases where different pathologies may require different surgical strategies, several other factors may contribute to treatment decision-making.

The occipital interhemispheric fissure has fewer bridging veins connecting the SSS than the parietal area. Anatomically important structures including the midbrain, internal cerebral veins, and veins of Rosenthal are able to be identified and avoided using the occipital interhemispheric fissure. Of course, attention must be paid to visual field defects caused by extravagant compression with the spatula on the medial surface of the occipital lobe. In addition to gentle manipulation of the occipital lobe, preservation of intact veins including cortical bridging veins and the internal occipital vein is crucial to avoiding postoperative brain edema and delayed hemorrhage. Compared with the prone position, the park bench position has proven effective in preventing excessive elevations in intrathoracic pressure causing the congestion of venous drainage from the head. With appropriate CSF release from either spinal or ventricular drainage, an occipital interhemispheric approach for splenial lesions might be more reasonable, even for abscesses.

\section{Conclusion}

This is the first report of a patient with bacterial abscess formation confined to the splenium of the corpus callosum. Treatment involved direct drainage via an occipital interhemispheric approach. Although extremely rare, brain abscess should be considered as a differential diagnosis for lesions in the splenium of the corpus callosum. Neuroradiologically, brain abscess in the splenium mimics the reversible transient focal change of the splenium occurring in association with mild encephalitis and metabolic disturbance. T2-weighted imaging and DWI demonstrate signal hyperintensity in both lesions. T1-weighted imaging after gadolinium administration may be useful to distinguish these pathologies, with enhancement of the capsule observed only in cases of abscess. An occipital interhemispheric approach to the splenium might be an important option even in cases of brain abscess, in place of stereotactic aspiration. 


\section{Acknowledgements}

This study was supported in part by research grants from the Takeda Science Foundation.

\section{Disclosure}

The authors report no conflict of interest concerning the materials or methods used in this study or the findings specified in this paper.

\section{References}

[1] Bottcher, J., Kunze, A., Kurrat, C., Schmidt, P., Hagemann, G., Witte, O.W., et al. (2005) Localized Reversible Reduction of Apparent Diffusion Coefficient in Transient Hypoglycemia-Induced Hemiparesis. Stroke, 36, e20-e22. http://dx.doi.org/10.1161/01.STR.0000155733.65215.c2

[2] Gurtler, S., Ebner, A., Tuxhorn, I., Ollech, I., Pohlmann-Eden, B. and Woermann, F.G. (2005) Transient Lesion in the Splenium of the Corpus Callosum and Antiepileptic Drug Withdrawal. Neurology, 65, 1032-1036. http://dx.doi.org/10.1212/01.wnl.0000179301.96652.27

[3] Kim, S.S., Chang, K.H., Kim, S.T., Suh, D.C., Cheon, J.E., Jeong, S.W., et al. (1999) Focal Lesion in the Splenium of the Corpus Callosum in Epileptic Patients: Antiepileptic Drug Toxicity? American Journal of Neuroradiology, 20, 125129.

[4] Loh, Y., Watson, W.D., Verma, A. and Krapiva, P. (2005) Restricted Diffusion of the Splenium in Acute Wernicke’s Encephalopathy. Journal of Neuroimaging: Official Journal of the American Society of Neuroimaging, 15, 373-375. http://dx.doi.org/10.1177/1051228405279037

[5] Maeda, M., Tsukahara, H., Terada, H., Nakaji, S., Nakamura, H., Oba, H., et al. (2006) Reversible Splenial Lesion with Restricted Diffusion in a Wide Spectrum of Diseases and Conditions. Journal of Neuroradiology, 33, 229-236. http://dx.doi.org/10.1016/S0150-9861(06)77268-6

[6] Fath-Ordoubadi, F., Lane, R.J. and Richards, P.G. (1997) Histological Surprise: Callosal Tuberculoma Presenting as Malignant Glioma. Journal of Neurology, Neurosurgery, and Psychiatry, 63, 98-99. http://dx.doi.org/10.1136/jnnp.63.1.98

[7] Lee, H.J., Williams, R., Kalnin, A. and Wolansky, L. (1996) Toxoplasmosis of the Corpus Callosum: Another Butterfly. American Journal of Roentgenology, 166, 1280-1281. http://dx.doi.org/10.2214/ajr.166.6.8633432

[8] Cox, J., Murtagh, F.R., Wilfong, A. and Brenner, J. (1992) Cerebral Aspergillosis: MR Imaging and Histopathologic Correlation. American Journal of Neuroradiology, 13, 1489-1492.

[9] Barlas, O., Sencer, A., Erkan, K., Eraksoy, H., Sencer, S. and Bayindir, C. (1999) Stereotactic Surgery in the Management of Brain Abscess. Surgical Neurology, 52, 404-410. http://dx.doi.org/10.1016/S0090-3019(99)00118-4

[10] Chang, Y.T., Lu, C.H., Chuang, M.J., Huang, C.R., Chuang, Y.C., Tsai, N.W., et al. (2010) Supratentorial DeepSeated Bacterial Brain Abscess in Adults: Clinical Characteristics and Therapeutic Outcomes. Acta Neurologica Taiwanica, 19, 178-183.

[11] Fuentes, S., Bouillot, P., Regis, J., Lena, G. and Choux, M. (2001) Management of Brain Stem Abscess. British Journal of Neurosurgery, 15, 57-62. http://dx.doi.org/10.1080/026886901300004120

[12] Moody, D.M., Bell, M.A. and Challa, V.R. (1988) The Corpus Callosum, a Unique White-Matter Tract: Anatomic Features That May Explain Sparing in Binswanger Disease and Resistance to Flow of Fluid Masses. American Journal of Neuroradiology, 9, 1051-1059.

[13] Rees, J.H., Smirniotopoulos, J.G., Jones, R.V. and Wong, K. (1996) Glioblastoma Multiforme: Radiologic-Pathologic Correlation. Radiographics, 16, 1413-1438.

[14] Erdag, N., Bhorade, R.M., Alberico, R.A., Yousuf, N. and Patel, M.R. (2001) Primary Lymphoma of the Central Nervous System: Typical and Atypical CT and MR Imaging Appearances. American Journal of Roentgenology, 176, 1319-1326. http://dx.doi.org/10.2214/ajr.176.5.1761319

[15] Demaerel, P., Van de Gaer, P., Wilms, G. and Baert, A.L. (1996) Interhemispheric Lipoma with Variable Callosal Dysgenesis: Relationship between Embryology, Morphology, and Symptomatology. European Radiology, 6, 904-909. http://dx.doi.org/10.1007/BF00240701

[16] Reinarz, S.J., Coffman, C.E., Smoker, W.R. and Godersky, J.C. (1988) MR Imaging of the Corpus Callosum: Normal and Pathologic Findings and Correlation with CT. American Journal of Roentgenology, 151, 791-798. http://dx.doi.org/10.2214/ajr.151.4.791

[17] DeLone, D.R., Goldstein, R.A., Petermann, G., Salamat, M.S., Miles, J.M., Knechtle, S.J., et al. (1999) Disseminated Aspergillosis Involving the Brain: Distribution and Imaging Characteristics. American Journal of Neuroradiology, 20, 
1597-1604.

[18] Winslow, H., Mickey, B. and Frohman, E.M. (2006) Sympathomimetic-Induced Kaleidoscopic Visual Illusion Associated with a Reversible Splenium Lesion. Archives of Neurology, 63, 135-137. http://dx.doi.org/10.1001/archneur.63.1.135

[19] Ogura, H., Takaoka, M., Kishi, M., Kimoto, M., Shimazu, T., Yoshioka, T., et al. (1998) Reversible MR Findings of Hemolytic Uremic Syndrome with Mild Encephalopathy. American Journal of Neuroradiology, 19, 1144-1145.

[20] Ho, M.L., Moonis, G., Ginat, D.T. and Eisenberg, R.L. (2013) Lesions of the Corpus Callosum. American Journal of Roentgenology, 200, W1-W16. http://dx.doi.org/10.2214/AJR.11.8080

[21] Oster, J., Doherty, C., Grant, P.E., Simon, M. and Cole, A.J. (2003) Diffusion-Weighted Imaging Abnormalities in the Splenium after Seizures. Epilepsia, 44, 852-854. http://dx.doi.org/10.1046/j.1528-1157.2003.40902.X

[22] Kimura, E., Okamoto, S., Uchida, Y., Hirahara, T., Ikeda, T., Hirano, T., et al. (2008) A Reversible Lesion of the Corpus Callosum Splenium with Adult Influenza-Associated Encephalitis/Encephalopathy: A Case Report. Journal of Medical Case Reports, 2, 220. http://dx.doi.org/10.1186/1752-1947-2-220

[23] Jang, Y.Y. and Lee, K.H. (2010) Transient Splenial Lesion of the Corpus Callosum in a Case of Benign Convulsion Associated with Rotaviral Gastroenteritis. Korean Journal of Pediatrics, 53, 859-862. http://dx.doi.org/10.3345/kjp.2010.53.9.859PMid:21189973

[24] Matsuoka, T., Yodoshi, T., Sugai, M., Hiyane, M., Matsuoka, T., Akeda, H., et al. (2013) A Case of Mild Encephalopathy with a Reversible Splenial Lesion Associated with G5P[6]Rotavirus Infection. Case Reports in Pediatrics, 2013, Article ID: 197163. http://dx.doi.org/10.1155/2013/197163

[25] Takanashi, J., Barkovich, A.J., Yamaguchi, K. and Kohno, Y. (2004) Influenza-Associated Encephalitis/Encephalopathy with a Reversible Lesion in the Splenium of the Corpus Callosum: A Case Report and Literature Review. American Journal of Neuroradiology, 25, 798-802.

[26] Conti, M., Salis, A., Urigo, C., Canalis, L., Frau, S. and Canalis, G.C. (2007) Transient Focal Lesion in the Splenium of the Corpus Callosum: MR Imaging with an Attempt to Clinical-Physiopathological Explanation and Review of the Literature. La Radiologia Medica, 112, 921-935. http://dx.doi.org/10.1007/s11547-007-0197-9

[27] Tada, H., Takanashi, J., Barkovich, A.J., Oba, H., Maeda, M., Tsukahara, H., et al. (2004) Clinically Mild Encephalitis/Encephalopathy with a Reversible Splenial Lesion. Neurology, 63, 1854-1858.

http://dx.doi.org/10.1212/01.WNL.0000144274.12174.CB 
Scientific Research Publishing (SCIRP) is one of the largest Open Access journal publishers. It is currently publishing more than 200 open access, online, peer-reviewed journals covering a wide range of academic disciplines. SCIRP serves the worldwide academic communities and contributes to the progress and application of science with its publication.

Other selected journals from SCIRP are listed as below. Submit your manuscript to us via either submit@scirp.org or Online Submission Portal.
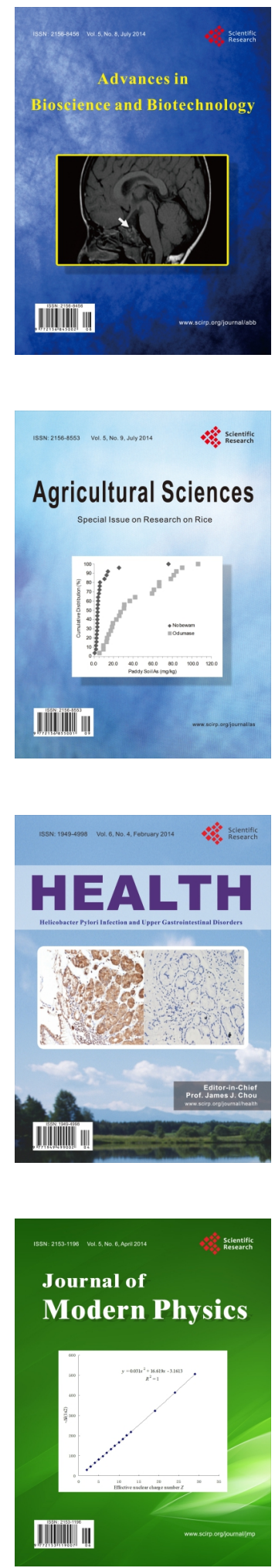
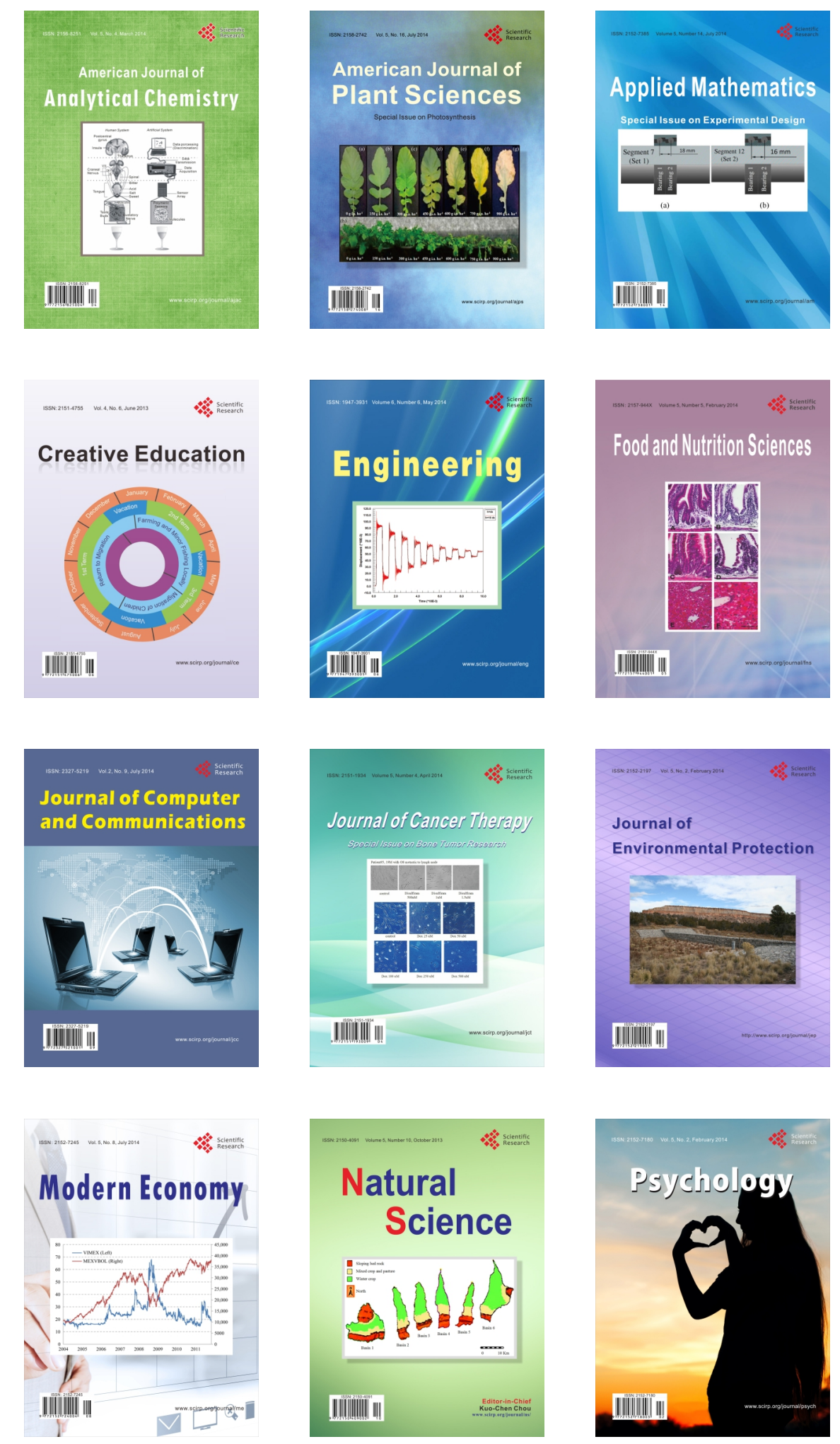